\title{
Supporting Operations Management Decisions with LP Parametric Analyses Using AIMMS
}

\author{
Imre Dimény ${ }^{1 *}$, Tamás Koltai ${ }^{1}$ \\ 1 Department of Management and Business Economics, Faculty of Economic and Social Sciences, Budapest University of \\ Technology and Economics, H-1521 Budapest, P.O.B. 91, Hungary \\ * Corresponding author, e-mail: dimeny@mvt.bme.hu
}

Received: 31 May 2019, Accepted: 22 February 2020, Published online: 08 June 2020

\begin{abstract}
Organizations all over the world use Business Analytics (BA) to gain insight in order to drive business strategy and planning. With the increasing amount of available data larger models are created to support decision making, but managers also must deal with the uncertainty of the input parameters. In this perspective Linear Programming (LP) models have two valuable properties: the required computation time allows large models to be solved and further valuable insight can be gained about the problem using sensitivity analysis. There is a wide range of tools available to solve LP problems. Many of these tools use an implementation of the simplex method and provides an optimal solution related sensitivity information. The sensitivity information generated by such solvers are often used by managers in the decision making process. There are situations when managers may have a hard time taking decision based on the information provided by most of the commercially available LP solvers. If the optimal solution of the primal problem (dual degeneracy) or the dual problem (primal degeneracy) is not unique, the resulting sensitivity information can be misleading for managers. In other cases, the resulted ranges may be too tight for decision support, thus information about a wider range is required. In this paper parametric analysis information is recommended to complete the traditional LP results in order to increase the insight of operations managers when using LP models for operation improvement.
\end{abstract}

Keywords

linear programming, sensitivity analyses, parametric analyses, AIMMS

\section{Introduction}

Organizations all over the world use Business Analytics (BA) to gain insight in order to drive business strategy and planning. With the increasing amount of available data, larger models are created to support decision making, but managers also must deal with the uncertainty of the input parameters. In this perspective LP models have two valuable properties: the required computation time allows large models to be solved and further valuable insight can be gained about the problem using sensitivity analysis. There is a wide range of available tools to solve LP problems. Many of these tools use an implementation of the simplex method and provide an optimal solution related to sensitivity information. The sensitivity information generated by such solvers are often used by managers in the decision making process.

Every linear programming problem, referred to as a primal problem, can be converted into a dual problem, which provides an upper bound to the optimal value of the primal problem. The optimal solution of an LP problem provides the optimal allocation of limited resources, while the optimal solution of the dual problem provides information about the marginal change of the objective function of the primal problem (shadow price), if a Right-HandSide (RHS) parameter changes.

Sensitivity analysis provides information about the validity range of the primal and dual optimum. The validity range of the Objective Function Coefficients (OFC) provides a range for each coefficient, within which the primal optimal solution will not change. Validity range of the RHS elements provides a range for each RHS element. Within this range the dual optimum will not change.

There are situations when managers may have a hard time taking a decision based on the information provided by most of the commercially available LP solvers. If the optimal solution of the primal problem (dual degeneracy) or the dual problem (primal degeneracy) is not unique, the resulting sensitivity information can be misleading for 
managers. In other cases, the resulted ranges may be too tight for decision support, thus information about a wider range is required.

The objective of this paper is to present a practical implementation of LP parametric analyses that provides correct LP sensitivity information even in the case of degeneracy and presents the objective value function for the whole feasible range of any required OFC or RHS parameter.

The paper is structured as follows: First, two problems related to the interpretation of the information provided by LP sensitivity analyses are presented. The first problem is degeneracy, and the second problem is the case of overly tight sensitivity ranges. Next, the objective value function is recommended to overcome the problems described and a possible practical implementation to calculate the objective value function is described. Finally, an AIMMS implementation and illustration example of the suggested method is presented.

\section{Interpretation problems of LP sensitivity analyses 2.1 Decision making under degeneracy}

Evans and Baker (1982) provided examples to show that under degeneracy the interpretation of sensitivity information calculated by most commercial LP solvers can be erroneous and have significant managerial implications. Problems and possible solutions related to LP Sensitivity analysis has an extensive literature since then.

Aucamp and Steinberg (1982) demonstrated that shadow prices are not necessarily equal to the dual variables, except in the case when the primal problem is nondegenerate, and suggested an alternative definition for the shadow price. Akgül (1984) separated the positive and the negative shadow prices. Gal (1986) made an extensive survey on the managerial interpretation of shadow prices. Many papers demonstrate erroneous management decisions based on the misinterpretation of sensitivity analysis results (see for example: Jansen et al., 1997; Rubin and Wagner, 1990).

The sensitivity information provided by commercially available solvers are correct from a mathematical point of view but may lead to erroneous decisions when used by managers. To differentiate between the mathematical and the managerial interpretation of sensitivity information Koltai and Terlaky (2000) classified three types of sensitivity information for which descriptive names were given by Hadigheh and Terlaky (2005).

- Type I (Basis Invariancy) is the traditional sensitivity analysis and determines those values of some RHS or OFC parameters for which a given optimal basis remains optimal.
- Type II (Support Set Invariancy) sensitivity determine invariant support set ranges of some parameters so that variables with a zero and with a positive value in the given optimal solution remain zero and positive in the optimal solution of the perturbed problem.

- Type III sensitivity (Optimal Partition Invariancy) returns ranges of some parameters for which the rate of change of the objective value function remains unchanged. Type III range of some parameters depends only on the problem data. The objective value function is a piecewise linear function of the parameter when the perturbation occurs in either the RHS or the OFC parameter of an LP model. The intervals, in which the rate of change of the optimal value function is constant, are sometimes referred to as the linearity intervals.

In non-degenerate cases the three types of sensitivities return identical ranges, but in a degenerate case different sensitivity information could be provided by LP solvers. Most of the commercial LP solvers provide only type I sensitivity information but from a management standpoint type III sensitivity information is far more important.

A practical approach to calculate type III sensitivity information was presented by Koltai and Tatay (2011). The suggested approach uses additional LP's to calculate the related sensitivity ranges.

\subsection{The problem of too tight sensitivity ranges}

To illustrate the problem of too tight sensitivity ranges three simple but similar problems are presented in Table 1.

The objective function and constraints and are identical for all the three problems, but LP2 has a different RHS parameter for constraint and LP3 has an additional constraint. The shadow price and the linearity interval related to the current value of the RHS parameter of constraint 2 are identical $(1.15,15)$ for all three LP problems. If the current value of a right-hand-side parameter is the result of an estimation or forecast - which is often the case - a value such close to the edge of the linearity interval is not a reliable information and requires further considerations.

The examination of the objective value function in a larger interval may help to evaluate the unreliability of the critical parameter. The change of the slope of the objective value function when leaving the upper limit of the linearity interval related to the RHS parameter of constraint 2 of LP1, LP2 and LP3 parameters illustrates the problem in Fig. 1. Bold values mark the current value and the related objective value, a continuous line marks the current 

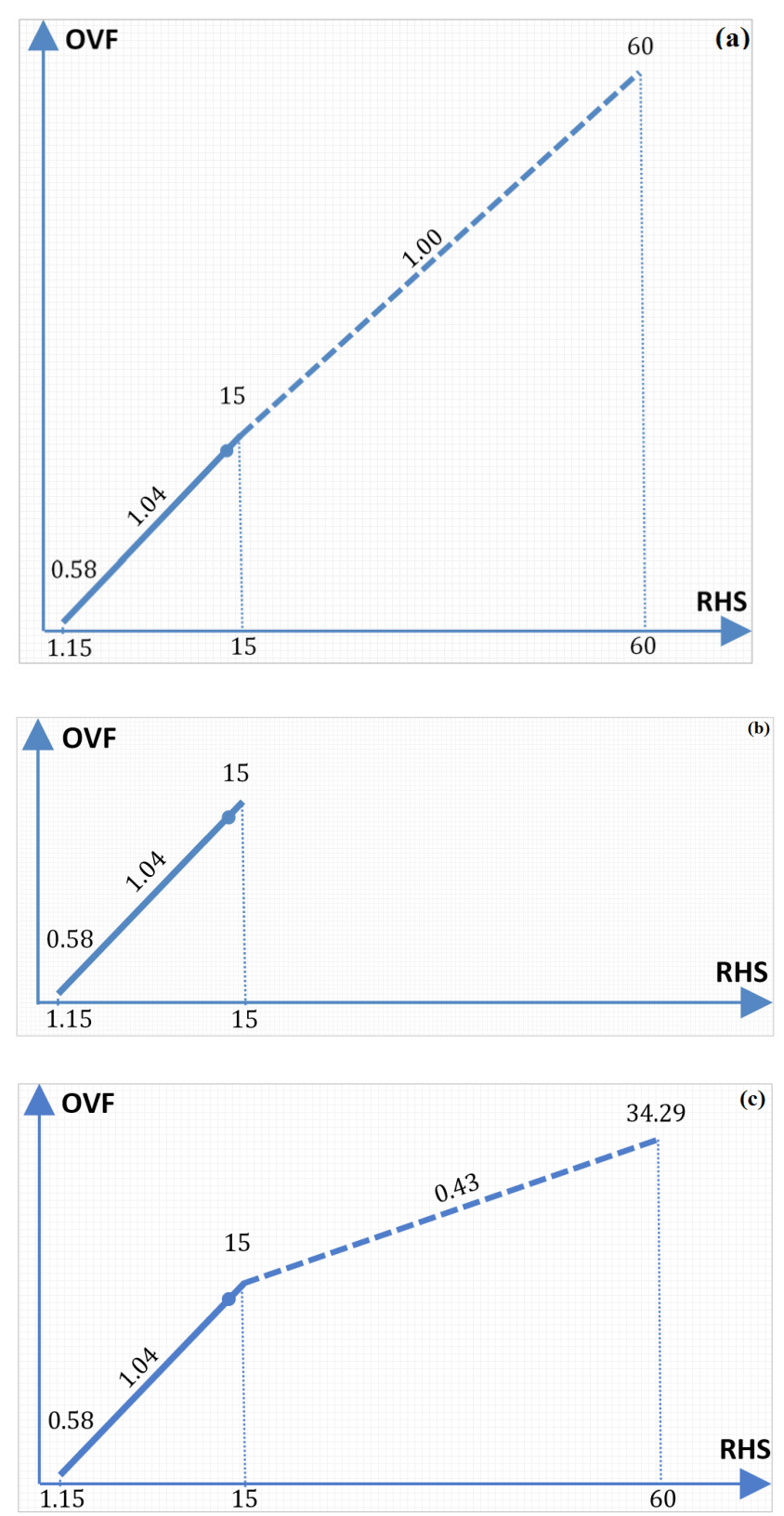

Fig. 1 (a) Objective value function related to the RHS parameter of constraint 2 for problem LP1; (b) Objective value function related to the RHS parameter of constraint 2 for problem LP2 (c) Objective value function related to the RHS parameter of constraint 2 for problem LP3 linearity interval and a dashed line marks other linearity interval of the objective value function if exists. The different evolution of objective value function requires different managerial actions.

The slope of the objective value related to constraint 2 in case of the LP1 problem is almost linear for a wide range. The slope changes from 1.04 to 1.00 when the RHS value of constraint 2 enters the next interval. In case of LP2 the problem becomes infeasible for values higher than the upper bound of the current linearity interval. Finally, in case of the LP3 problem the slope of the objective value function related to the same RHS parameter changes significantly (from 1.04 to 0.43 ).

Similarly, the visualization of the objective value function related to some OFC parameters could further clarify the different decision making context.

The parameters of problems LP4 and LP5 presented in Table 1, are identical except of the RHS parameter related to constraint 2. Fig. 2 (a) shows the objective value function related to the objective function coefficient of the $x_{2}$ variable for problem LP4 while Fig. 2 (b) shows the objective value function for the objective function coefficient of the $x_{2}$ variable for problem LP5.

In case of the LP4 problem, the slope of the objective value function related to variable $x_{2}$ has a minor modification when leaving the linearity interval to right. In case of the LP5 problem, increasing the OFC parameter related to variable $x_{2}$ above the upper limit of its validity range, the optimal value is no longer 0 . This means that not only will the quantities change, but also the structure of the plan.

Based on the two illustrations presented, it can be concluded that evolution of the objective value function in a wider domain of the critical parameter may help the decision maker.

\section{Objective value function}

The development of the mathematical modelling tools and the increasing computational power of commercially available computers makes it possible to map the effect

Table 1 Illustration LP problems

\begin{tabular}{|c|c|c|c|c|c|}
\hline & LP1 & LP2 & LP3 & LP4 & LP5 \\
\hline Objective & $\max \left(x_{1}+x_{2}+x_{3}\right)$ & $\max \left(x_{1}+x_{2}+x_{3}\right)$ & $\max \left(x_{1}+x_{2}+x_{3}\right)$ & $\max \left(x_{1}+x_{2}+x_{3}\right)$ & $\max \left(x_{1}+x_{2}+x_{3}\right)$ \\
\hline Constraint 1 & $x_{1}+26 x_{2}+x_{3} \geq 15$ & $x_{1}+26 x_{2}+x_{3} \geq 15$ & $x_{1}+26 x_{2}+x_{3} \geq 15$ & $x_{1}+2 x_{2}+x_{3} \leq 10$ & $x_{1}+2 x_{2}+x_{3} \leq 10$ \\
\hline Constraint 2 & $x_{1}+2 x_{2}+x_{3}=14.5$ & $x_{1}+2 x_{2}+x_{3}=14.5$ & $x_{1}+2 x_{2}+x_{3}=14.5$ & $x_{1}+x_{3} \leq 3$ & $x_{1}+x_{3} \leq 10$ \\
\hline Constraint 3 & $x_{1}+2 x_{2}+x_{3} \leq 60$ & $x_{1}+2 x_{2}+x_{3} \leq 15$ & $x_{1}+2 x_{2}+x_{3} \leq 60$ & & \\
\hline Constraint 4 & & & $4 x_{1}+x_{2}+4 x_{3} \leq 60$ & & \\
\hline Other constraints & $x_{1}, x_{2}, x_{3} \geq 0$ & $x_{1}, x_{2}, x_{3} \geq 0$ & $x_{1}, x_{2}, x_{3} \geq 0$ & $x_{1}, x_{2}, x_{3} \geq 0$ & $x_{1}, x_{2}, x_{3} \geq 0$ \\
\hline
\end{tabular}



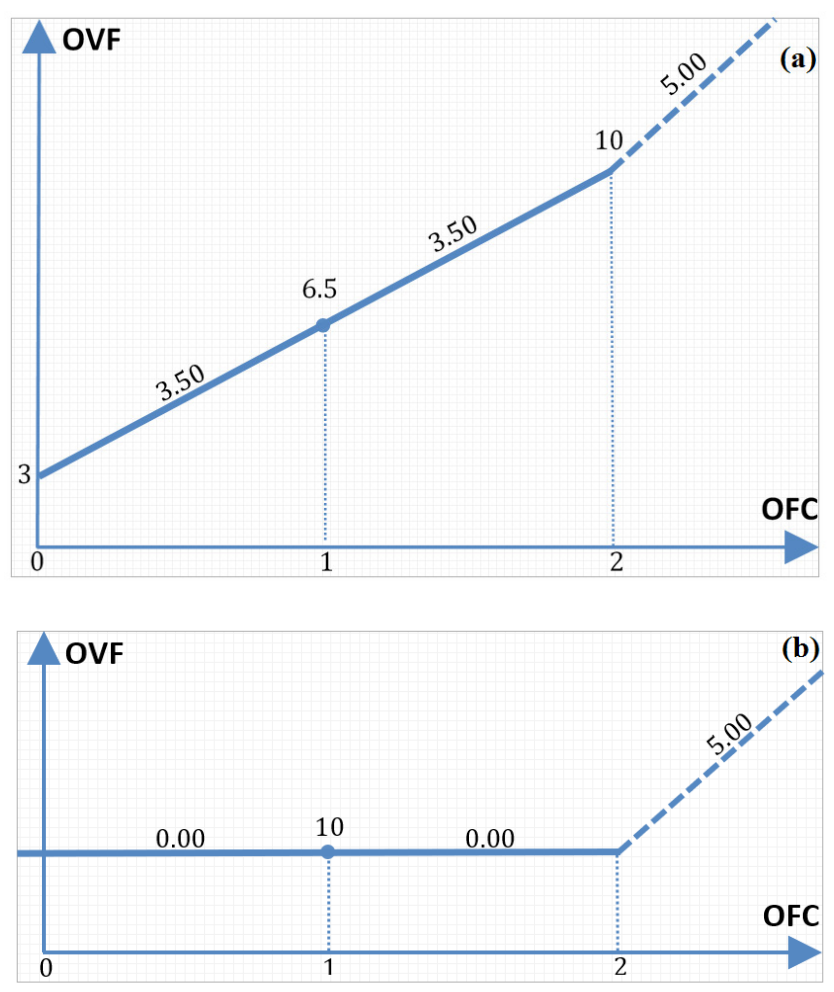

Fig. 2 (a) Objective value function related to the OFC parameter of the variable for problem LP4; (b) Objective value function related to the OFC parameter of the variable for problem LP5

of changes of the OFC and RHS parameters for the whole feasible/bounded region.

This map could give managers a complete overview on how the change of some critical parameters affects the objective value of the LP model and could give valuable additional information when sensitivity ranges provided by the LP solvers are overly tight.

\subsection{Domain of the objective value function}

The domain set of the objective value function of some OFC or RHS parameters contains those values for which the related perturbed LP problem is feasible and bounded.

The modification of an OFC parameter does not influence the feasibility of the LP problem, however, after a certain point, the previously bounded problem may become unbounded. In this case the domain of the related objective value function will become bounded in one direction. In any other cases the domain of an objective value function related to an OFC parameter will be a set of all real numbers, with infinite linearity intervals at both ends. Change on the RHS parameter in one direction decreases the feasible region of the LP problem, while changes on the opposite direction expands the feasible region while the corresponding constraint is active. Once the corresponding constraint is no more active the related shadow price will turn to 0 and the length of this last linearity interval will be infinite.

To create the objective value function related to one of the OFC or RHS parameters a set of consecutive intervals $\left(I^{k}, k=1 . . K\right)$ with constant rate of change of the objective value function within each interval must be defined. For each interval the rate of change of the objective function $\left(I_{\text {rate }}^{k}\right)$ and the value of the objective function at the starting and ending points must be calculated ( $I_{s}^{k}$ and $I_{e}^{k}$ ).

\subsection{Initial steps to calculate the objective value function} Consider the $\max \left(\mathbf{c}^{T} \mathbf{x}\right), \mathbf{A x} \leq \mathbf{b}, \mathbf{x} \geq 0$ standard form of the linear program (Hillier and Lieberman, 1995). The elements of the $\mathbf{c} \mathrm{OFC}$ vector are $c_{1}, c_{2}, \ldots, c_{3}$, furthermore, the elements of the $b$ RHS parameters vector are $b_{1}, b_{2}, \ldots, b_{3}$.

As an initial step, a parametric model for solving the original LP with modified RHS or OFC values must be implemented. Let $L P(\lambda \leftarrow v)$ note a modified version of the original LP where parameter $\lambda$ is modified to $v$ and let $O F^{*}(\lambda \leftarrow v)$ be the corresponding optimal objective value of the modified LP.

For the calculation of the correct sensitivity ranges even in the degenerate case, a parametrized version of the additional LPs described by Koltai and Tatay (2011) must also be implemented.

Type III sensitivity provides information about the invariance of the rate of change of the objective value function. Let $\xi_{j}^{+}\left(b^{\prime}\right)$ and $\xi_{j}^{-}\left(b^{\prime}\right)$ denote the maximal increase and the maximal decrease allowed for the $b^{\prime}$ right-handside parameter of constraint $j$ to remain within the type III invariancy interval related to the modified $L P\left(b_{j} \leftarrow b^{\prime}\right)$ problem.

Similarly let $\gamma_{i}^{+}\left(c^{\prime}\right)$ and $\gamma_{i}^{-}\left(c^{\prime}\right)$ denote the maximal increase and the maximal decrease allowed for the $c^{\prime}$ objective function coefficient of variable $i$ to remain within the type III invariancy interval related to the modified $L P\left(c_{i} \leftarrow c^{\prime}\right)$ problem.

\subsection{Algorithm for calculating the consecutive RHS intervals}

Intervals are calculated starting from the original RHS parameter separately for increasing and for decreasing directions and only when the original LP has at least one feasible solution.

As an initial step, the maximal feasible change of the RHS parameter in the selected direction that allows the LP problem to remain feasible must be calculated. To calculate 
these values, an additional LP must be solved for each RHS parameter and to each direction. The maximal increase as well as the maximal decrease are nonnegative numbers. In the additional LP's, the constraint related to the selected RHS parameter becomes the objective value function. Assume that $\beta_{j}^{+}$and $\beta_{j}^{-}$are the maximal feasible increase and the maximal feasible decrease of the RHS parameter related to constraint $j$. One of these values may be infinite.

The rate of change denoted by $S P^{+}$and $S P^{-}$are the right and left shadow prices of the modified $L P\left(b_{j} \leftarrow b_{j}^{k}\right)$ problem where $b_{j}^{k}$ is the RHS parameter of constraint $j$ when calculating interval $k$.

In the initialization step the maximal feasible modification of the RHS parameter is calculated. Next, the following steps are repeated until the calculated maximal feasible modification is reached, or the maximum increase/ decrease is infinite:

- create and solve the modified $L P\left(b_{j} \leftarrow b_{j}^{k}\right)$ problem,

- calculate the type III range for the required direction, $\left(\xi_{j}^{+}\left(b_{j}^{k}\right)\right.$ and $\xi_{j}^{-}\left(b_{j}^{k}\right)$ respectively),

- collect interval data and shadow price:

- $\left(b_{j}^{k}, I_{s}^{k}+\xi_{j}^{+}\left(b_{j}^{k}\right)\right)$ and $S P^{+}$for increasing intervals, $\left(I_{e}^{k}-\xi_{j}^{-}\left(b_{j}^{k}\right), b_{j}^{k}\right)$ and $S P^{-}$for decreasing intervals,

- $\quad$ set $b_{j}^{k+1}=I_{e}^{k-1}$ for increasing intervals and $b_{j}^{k+1}=I_{s}^{k-1}$ for decreasing intervals.

\subsection{Algorithm for calculating the consecutive OFC intervals}

The modification of an OFC parameter does not influence the feasibility of the LP problem, however, after a certain point, the previously bounded problem may become unbounded.

In case of OFC parameters, the objective value is changed exclusively by the change of the OFC parameter and the rate of change is equal to the value of variable $i$ in the optimal solution $\left(x_{i}\right)$ of the modified $L P\left(c_{i} \leftarrow c_{i}^{k}\right)$ problem where $c_{i}^{k}$ is the OFC parameter related to variable $i$ when calculating interval $k$.

The following steps are repeated while the maximum increase/decrease is finite:

- create and solve the modified $L P\left(c_{i} \leftarrow c_{i}^{k}\right)$ problem,

- calculate type III range for the required direction ( $\gamma_{i}^{+}\left(c_{i}^{k}\right)$ and $\gamma_{i}^{-}\left(c_{i}^{k}\right)$ respectively),

- collect interval data and optimal value:
- $\left(c_{i}^{k}, I_{s}^{k}+\gamma_{i}^{+}\left(c_{i}^{k}\right)\right)$ for increasing intervals and $\left(I_{e}^{k}-\gamma_{i}^{-}\left(c_{i}^{k}\right), c_{i}^{k}\right)$ for decreasing intervals and $I_{\text {rate }}^{k}=x_{i}$,

- $\operatorname{set} c_{i}^{k+1}=I_{e}^{k}$ for increasing intervals and $c_{i}^{k+1}=I_{s}^{k}$ for decreasing intervals.

\section{AIMMS implementation}

From a practical point of view, creating a tool that can calculate and visualize the parametric objective value function requires a good solver, good algorithmic capabilities and graphical user interface editing option. Such a tool is provided by the AIMMS Platform, which is often used for solving commercial optimization problems in a wide range of industries including retail, consumer products, healthcare, oil and chemicals, steel production and agribusiness (Roelofs and Bisschop, 2018).

AIMMS Prescriptive Analytics Platform offers a straightforward mathematical modelling environment and a wide range of available solvers. AIMMS also features an advanced graphical user interface editor which allows the creation of optimization application to individuals without a technical or analytics background.

AIMMS' own structural language allows the creation of procedures which connect the multiple models required to calculate the type III ranges for all the parameters.

The list of available solvers in AIMMS also includes simplex method-based solvers such as CPLEX which can be instructed to use an initial solution and this way the calculation time can be significantly decreased. (Dimény and Koltai, 2018)

Since many of the OFC and RHS parameters are related to technical constraints and not relevant for managerial decision, the computation time can be decreased if irrelevant parameters are left out of calculation. With the use of the build-in user interface editor, a user-friendly interface can help the decision makers for selecting the relevant parameters of the model.

AIMMS version 4.42 was used to create the required mathematical models, implement the algorithms and create simple user interface, while CPLEX version 12.7.1 was used to solve the generated LP problems and obtain type I sensitivity information. Four parametrized models were created in AIMMS: 
- general parametrized linear program to solve the $L P\left(c_{i} \leftarrow c_{i}^{k}\right)$ and the $L P\left(b_{j} \leftarrow b_{j}^{k}\right)$ problems,

- modified parametrized linear program to calculate the maximal feasible increase/decrease of the RHS parameters,

- parametrized linear program to calculate the type III ranges of the RHS parameters,

- parametrized linear program to calculate the type III ranges of the $\mathrm{OFC}$ parameters.

To collect all type III intervals, the implementation of the algorithms presented previously are needed. The algorithm collects the data related to the intervals, which can then be visualized using tables and line charts on pages created with the AIMMS user interface editor. The implemented solution consists of the following pages:

- LP definition page is the input page, where the desired LP can be formulated.

- LP solution page contains the original results provided by the solver.

- Two pages for presenting the RHS interval data using table and line charts.

- Two pages for presenting the OFC interval data using table and line charts.

\section{Illustration example}

To illustrate the benefits of having type I, type III or the complete OVF range available, a production planning problem taken from Nahmias (1993) is presented. The problem was used by several earlier papers to demonstrate different approaches to LP sensitivity analysis (Borgonovo et al., 2018; Koltai and Tatay, 2011). The objective of the problem is to find the lowest cost production plan for 6 months. Production quantity can be influenced by hiring or firing workers and excess production is kept in inventory.

Let $h_{t}$ denote the cost of hiring, $f_{t}$ the cost of firing, $i_{t}$ the inventory holding cost in period $t(t=1, \ldots, 6)$. Decision variables $W_{t}, H_{t}, F_{t}, P_{t}, I_{t}$ denote the workforce, hired workers, fired workers, production and inventory for period $t$, respectly. An initial $W_{0}$ workforce and $I_{0}$ inventory level and $I_{6}$ closing inventory level is assumed. Demand for period $t$ is denoted by $D_{t}$ and the value of $D_{2}$ is set to 552 instead of 640 of the original problem, to make the LP degenerate. The relation of workforce and productivity is expressed using the $K=0.1465$ productivity constant.

The optimal production plan can be calculated using the following LP: $\min \left(\sum_{t=1}^{6} h_{t} H_{t}+\sum_{t=1}^{6} f_{t} F_{t}+\sum_{t=1}^{6} i_{t} I_{t}\right)$

subject to

$W_{t}-W_{t-1}-H_{t}+F_{t}=0, t=1 . .6$

$P_{t}-I_{t}+I_{t-1}=D_{t}, t=1 . .6$

$P_{t}-K n_{t} W_{t}=0, t=1 . .6$

$W_{0}=300$

$I_{0}=500$

$I_{6}=600$

$K=0.1465$

Fig. 3 contains part of the AIMMS page displaying the parameters of the model, the optimal solution, furthermore, the shadow price and validity range information of the OFC and RHS parameters.

The problem is solved as a continuous variable problem and according to the optimal solution 34 workers must be fired in period 1 and hiring of 472 workers is required in period 5. With the modified demand for period 2, became dual degenerate. The optimal solution of the dual problem is non-unique which leads to non-unique shadow prices for some of the RHS parameters of the primal problem.

For the RHS element $d_{1}$ the shadow price calculated by the solver is -24.18 while the validity range of the shadow price is $(-2152,1280)$. Due to the degeneracy of the optimal solution, the shadow price and validity interval calculated by the solver provides only information about the consequences of a decrease in demand for this period.

The consequences of a small increase in demand on the objective value function are provided by type III sensitivity range and the right shadow price. This could suggest to managers that an increase in demand is not desirable. The complete objective value function provides a much better insight for the decision maker. The increase in demand by more than $12 \%$ increases the objective value and further increase in demand will increase the objective value even more. In this case even the type III information could mislead managers.

Fig. 4 displays the objective value function related to the RHS parameter $d_{1}$. Note that the domain of the objective value function may contain negative demand values as well which, in most of the cases, are irrelevant from a management stand point. 


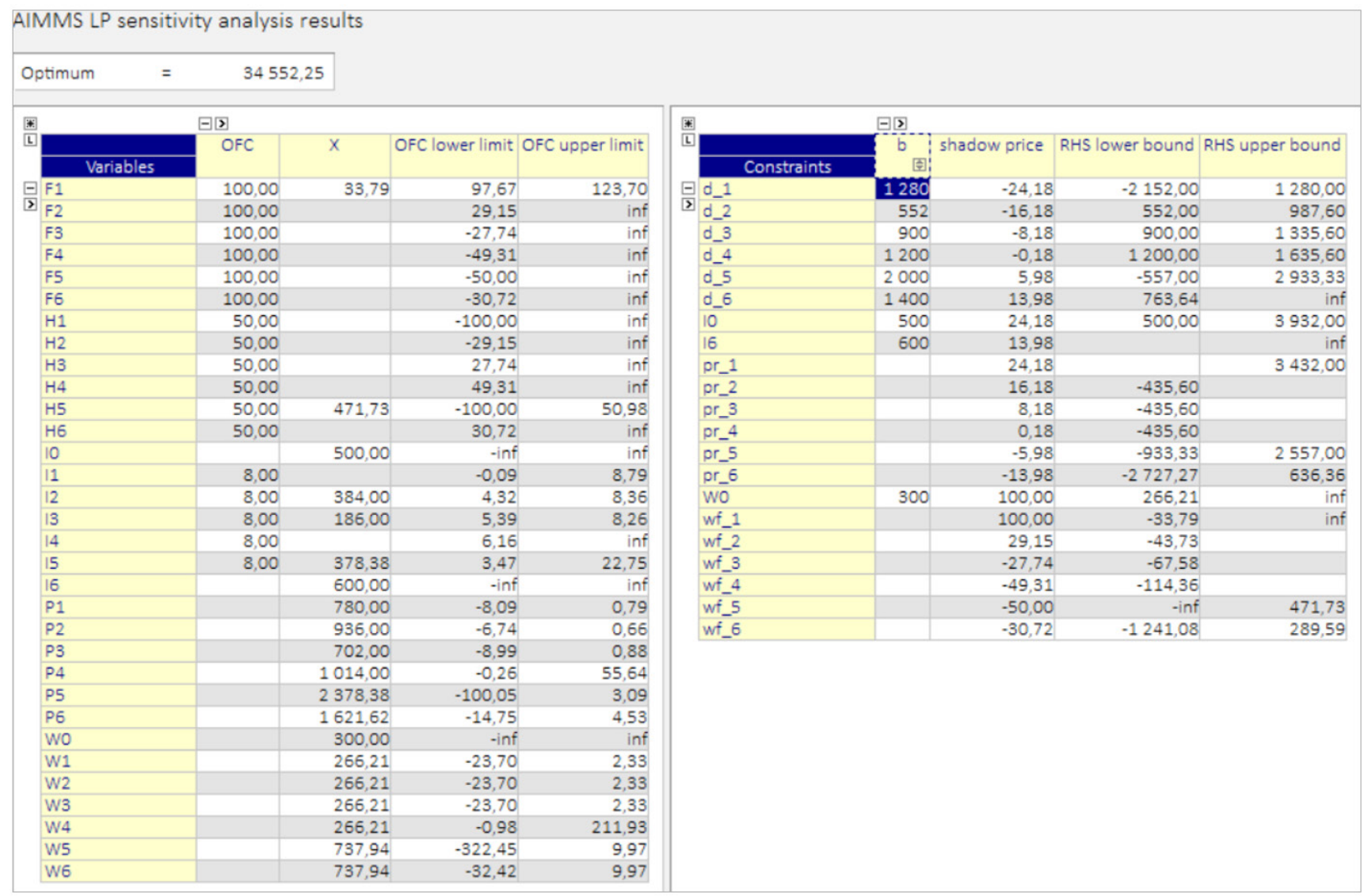

Fig. 3 Part of the AIMMS page displaying the optimal solution, shadow price and validity ranges related to the OFC and RHS parameters as calculated by CPLEX

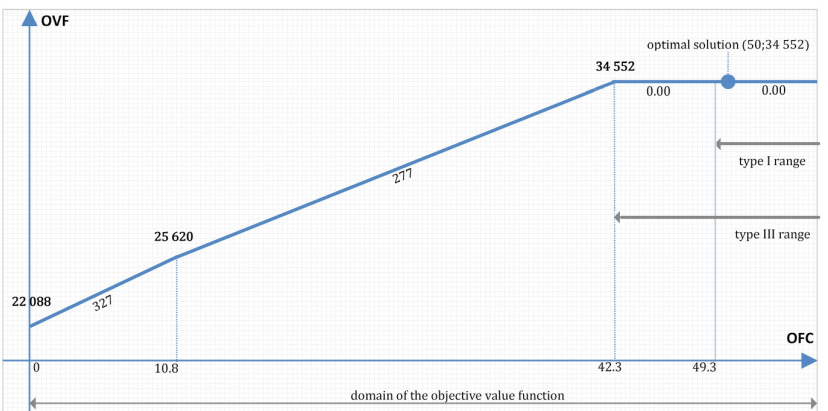

Fig. 4 Objective value function related to positive values of the RHS parameter

Fig. 5 displays the objective value function related to the OFC parameter $h_{4}$ and shows the consequences of the change in the hiring cost. The figure presents the difference between the domains of the type I range, type III range and the objective value function. In this case the left edge of the type III interval is smaller than the left edge of the type I range.

It must be noted that the domain of this function is left bounded. For $h_{4}<-100$ the problem becomes unbounded. In this case the cost of firing would be smaller than the gain of hiring and the objective value could be increased to infinity by hiring and firing unlimited amount of workforce.

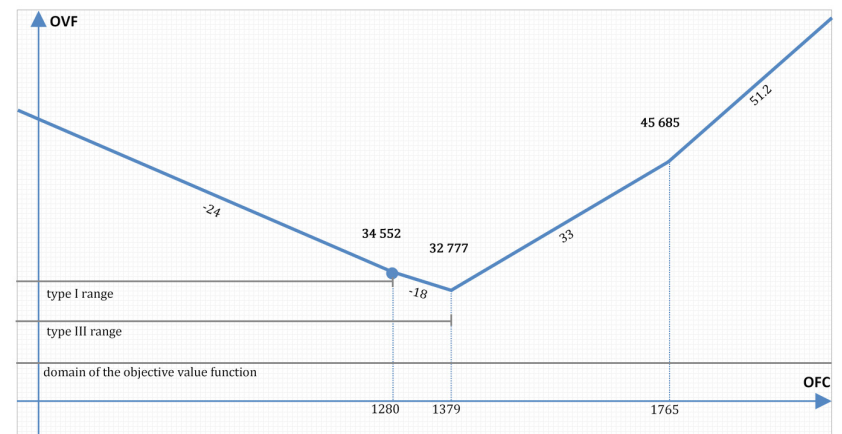

Fig. 5 Objective value function related to the OFC parameter

Fig. 6 and Fig. 7 shows the results of the OFC and RHS parametric analyses results created by the AIMMS application.

The figures contain the interval start and end points, the rate of change of the objective value function within the interval, the value of the objective value function at the start and end point and the optimal solution related.

Compared with the results provided by the basic AIMMS solver, it can be noticed that the solver calculated misleading information about the validity intervals. The misleading information is the consequence of degeneracy. The presented method provides correct LP sensitivity information 


\begin{tabular}{|c|c|c|c|c|c|c|c|c|}
\hline \multicolumn{9}{|c|}{ RHS sensitivity analysis results } \\
\hline \multirow{2}{*}{\multicolumn{9}{|c|}{ 娄四 }} \\
\hline \multirow{2}{*}{\multicolumn{9}{|c|}{\begin{tabular}{|l|l|} 
- Constraints $\mathrm{T}$ & Intervals \\
\end{tabular}}} \\
\hline & & & & & & & & \\
\hline \multirow{5}{*}{$\nabla$} & d_1 & $\square$ & 0 & $-6152,00$ & $-2152,00$ & $-26,02$ & 221616,00 & 117539,99 \\
\hline & & & 1 & $-2152,00$ & 1280,00 & $-24,18$ & 117539,99 & 34552,25 \\
\hline & & & 2 & 1280,00 & 1379,00 & $-17,93$ & 34552,25 & 32777,19 \\
\hline & & & 3 & 1379,00 & 1767,05 & 33,26 & 32777,19 & 45685,44 \\
\hline & & & 4 & 1767,05 & inf & 51,19 & 45685,44 & inf \\
\hline \multirow{10}{*}{\multicolumn{2}{|c|}{ d_2 }} & $\nabla$ & 0 & $-6100,00$ & $-2005,00$ & $-21,43$ & 168400,00 & 80626,84 \\
\hline & & & 1 & $-2005,00$ & 552,00 & $-18,02$ & 80626,84 & 34552,25 \\
\hline & & & 2 & 552,00 & 987,60 & $-16,18$ & 34552,25 & 27503,99 \\
\hline & & & 3 & 987,60 & 1044,90 & $-16,00$ & 27503,99 & 26587,19 \\
\hline & & & 4 & 1044,90 & 1084,57 & $-8,00$ & 26587,19 & 26269,84 \\
\hline & & & 5 & 1084,57 & 1153,80 & $-5,08$ & 26269,84 & 25917,89 \\
\hline & & & 6 & 1153,80 & 1299,00 & & 25917,89 & 25917,89 \\
\hline & & & 7 & 1299,00 & 1420,00 & 8,00 & 25917,89 & 26885,89 \\
\hline & & & 8 & 1420,00 & 2533,58 & 8,93 & 26885,89 & 36828,04 \\
\hline & & & 9 & 2533,58 & inf & 26,91 & 36828,04 & inf \\
\hline \multirow{10}{*}{\multicolumn{2}{|c|}{ d_3 }} & $\nabla$ & 0 & $-5200,00$ & $-3337,00$ & $-19,79$ & 119600,00 & 82740,34 \\
\hline & & & 1 & $-3337,00$ & $-1657,00$ & $-13,43$ & 82740,34 & 60170,84 \\
\hline & & & 2 & $-1657,00$ & 900,00 & $-10,02$ & 60170,84 & 34552,25 \\
\hline & & & 3 & 900,00 & 1335,60 & $-8,18$ & 34552,25 & 30988,79 \\
\hline & & & 4 & 1335,60 & 1392,90 & $-8,00$ & 30988,79 & 30530,39 \\
\hline & & & 5 & 1392,90 & 1432,57 & & 30530,39 & 30530,39 \\
\hline & & & 6 & 1432,57 & 2087,51 & 2,92 & 30530,39 & 32440,43 \\
\hline & & & 7 & 2087,51 & 3013,67 & 8,00 & 32440,43 & 39849,65 \\
\hline & & & 8 & 3013,67 & 3785,46 & 16,00 & 39849,65 & 52198,35 \\
\hline & & & 9 & 3785,46 & inf & 24,77 & 52198,35 & inf \\
\hline \multirow{8}{*}{\multicolumn{2}{|c|}{ d_4 }} & $\nabla$ & 0 & $-4000,00$ & $-1822,00$ & $-17,44$ & 80221,71 & 42241,76 \\
\hline & & & 1 & $-1822,00$ & $-1357,00$ & $-5,43$ & 42241,76 & 39714,84 \\
\hline & & & 2 & $-1357,00$ & 1200,00 & $-2,02$ & 39714,84 & 34552,25 \\
\hline & & & 3 & 1200,00 & 1635,60 & $-0,18$ & 34552,25 & 34473,59 \\
\hline & & & 4 & 1635,60 & 3303,71 & & 34473,59 & 34473,59 \\
\hline & & & 5 & 3303,71 & 4458,56 & 8,00 & 34473,59 & 43712,36 \\
\hline & & & 6 & 4458,56 & 5998,35 & 16,00 & 43712,36 & 68349,07 \\
\hline & & & 7 & 5998,35 & inf & 22,82 & 68349,07 & inf \\
\hline \multirow{7}{*}{\multicolumn{2}{|c|}{$d_{2}$}} & $\nabla$ & 0 & $-2000,00$ & $-557,00$ & $-21,69$ & 50560,00 & 19258,84 \\
\hline & & & 1 & $-557,00$ & 2000,00 & 5,98 & 19258,84 & 34552,25 \\
\hline & & & 2 & 2000,00 & 2933,33 & 5,98 & 34552,25 & 40134,52 \\
\hline & & & 3 & 2933,33 & 3368,93 & 7,82 & 40134,52 & 43540,66 \\
\hline & & & 4 & 3368,93 & 5692,90 & 8,00 & 43540,66 & 62132,39 \\
\hline & & & 5 & 5692,90 & 7301,80 & 16,00 & 62132,39 & 87874,79 \\
\hline & & & 6 & 7301,80 & inf & 23,03 & 87874,79 & \\
\hline
\end{tabular}

Fig. 6 AIMMS page with table containing the OFC intervals

in case of degeneracy as well. Furthermore, the implemented AIMMS algorithm presents the objective value function for the whole feasible range of any required objective function coefficient and right-hand-side parameter.

\section{Conclusion}

In this paper, the implementation of parametric analysis of LP models in AIMMS to support operations management decision making is presented. The information provided by 
OFC sensitivity analysis results

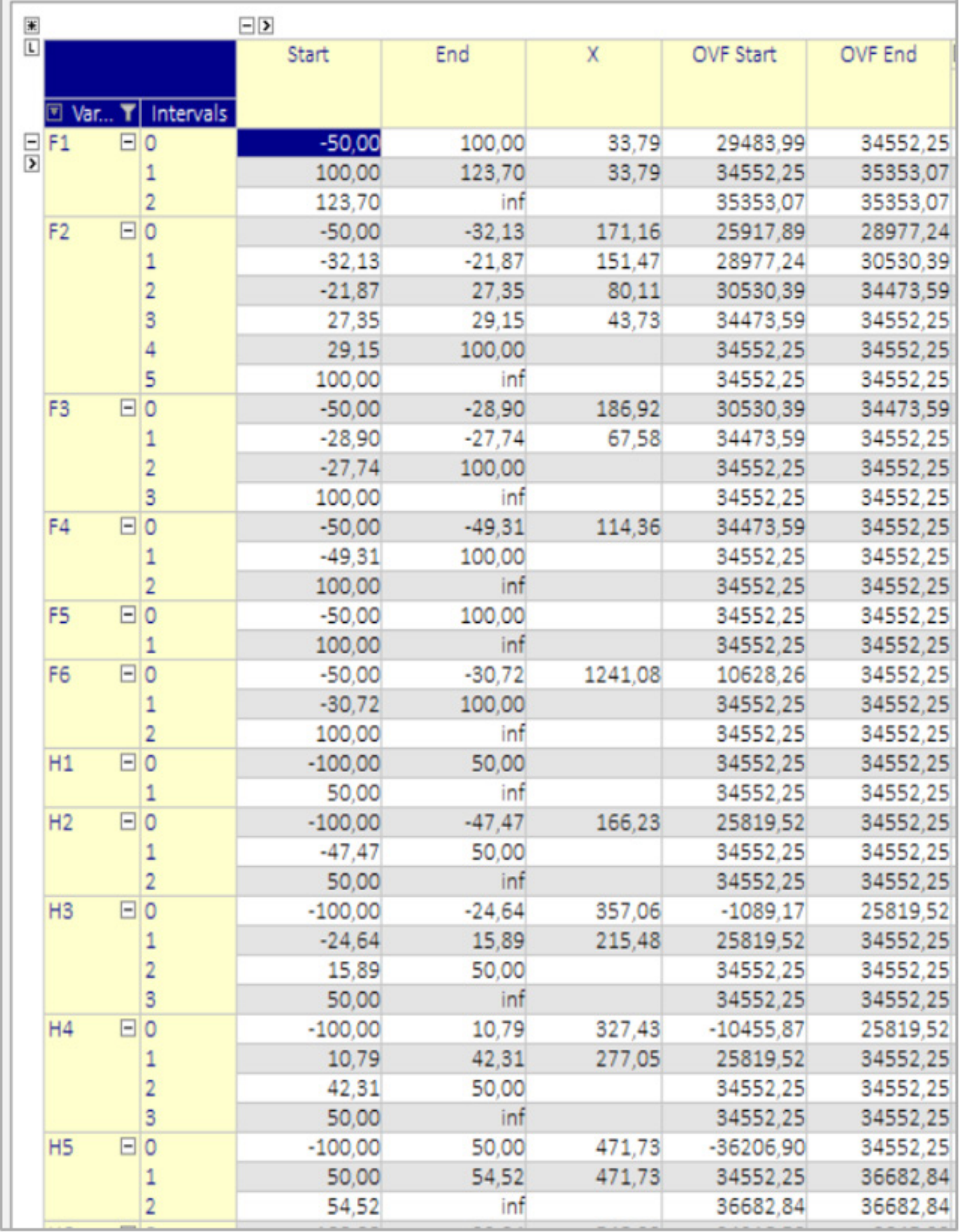


the suggested method describes the objective value function in the feasible range of any OFC and RHS parameters.

The benefits of this information are twofold:

Since all values of the objective value function are known, there are no misleading results as a consequence of degeneracy. If appropriate, the left and right shadow prices (slope of the objective value function in case of decrease and increase) are given, and the correct ranges of all parameters are calculated.

The traditional sensitivity ranges provided by most commercial LP solvers are completed with further information. In our case, the effect of the parameter change is known, not only in the close neighborhood of the original value, but also in the whole feasible region.

Compared to parametric linear programming, whose theory is described by Nozicka et al. (1974), although the method presented in this paper will result the same objective value function, two difference has to be noted:

- the focus of the presented method is on finding linearity intervals of the objective value function instead of ranges of some parameters for which the optimal basis remains optimal, and therefore less iteration is needed to construct the objective value function

\section{References}

Akgül, M. (1984) "A Note on Shadow Prices in Linear Programming", Journal of the Operational Research Society, 35(5), pp. 425-431. https://doi.org/10.1057/jors.1984.83

Aucamp, D. C., Steinberg, D. I. (1982) "The Computation of Shadow Prices in Linear Programming", Journal of the Operational Research Society, 33(6), pp. 557-565. https://doi.org/10.1057/jors.1982.118

Borgonovo, E., Buzzard, G. T., Wendell, R. E. (2018) "A global tolerance approach to sensitivity analysis in linear programming", European Journal of Operational Research, 267(1), pp. 321-337. https://doi.org/10.1016/j.ejor.2017.11.034

Dimény, I., Koltai, T. (2018) "Some practical issues related to the implementation of type III sensitivity analysis of LP models", In: 8th VOCAL Optimization Conference: Advanced Algorithms, Esztergom, Hungary, pp. 32-37.

Evans, J. R., Baker, N. R. (1982) "Degeneracy and the (Mis)interpretation of Sensitivity Analysis in Linear Programming", Decision Sciences, 13(2), pp. 348-354. https://doi.org/10.1111/j.1540-5915.1982.tb00155.x

Gal, T. (1986) "Shadow prices and sensitivity analysis in linear programming under degeneracy", Operations-Research-Spektrum, 8(2), pp. 59-71. https://doi.org/10.1007/BF01719736

Hillier, F. S., Lieberman, G. J. (1995) "Introduction to Operation Research", McGraw-Hill, New York, NY, USA.
- the presented method completely reveals the objective value function with its largest domain set for which the problem is feasible and bounded.

The method can be used to support OM decision whenever the problem of the allocation of scarce resources must be solved, and LP models can properly describe or approximate the problem. The created AIMMS application, beside showing the consecutive type III intervals in a table format, also contains a graphical presentation of the results, to create a better overview of the decision situation. The presented objective value function of any of the critical parameters can help operation managers to see directly the effect of planned or random parameter changes, or the possible consequences of the inaccuracy of data applied in the operation planning phase.

\section{Acknowledgement}

The research reported in this paper has been supported by the National Research, Development and Innovation Fund (TUDFO/51757/2019-ITM, Thematic Excellence Program).

Jansen, B., de Jong, J. J., Roos, C., Terlaky, T. (1997) "Sensitivity analysis in linear programming: just be careful!", European Journal of Operational Research, 101(1), pp. 15-28. https://doi.org/10.1016/S0377-2217(96)00172-5

Koltai, T., Tatay, V. (2011) "A practical approach to sensitivity analysis in linear programming under degeneracy for management decision making", International Journal of Production Economics, 131(1), pp. 392-398.

https://doi.org/10.1016/j.ijpe.2010.04.037

Koltai, T., Terlaky, T. (2000) "The difference between the managerial and mathematical interpretation of sensitivity analysis results in linear programming", International Journal of Production Economics, 65(3), pp. 257-274

https://doi.org/10.1016/S0925-5273(99)00036-5

Nahmias, S. (1993) "Production and Operations Analysis", Irwin, Burr Ridge, IL, USA.

Nozicka, F., Guddat, J., Hollatz, H., Bank, B. (1974) "Theorie der Linearen Parametrische Optimierung" (Theory of Linear Parametric Optimization), Akademie Verlag, Berlin, Germany. (in German)

Roelofs, M., Bisschop, J (2018) "AIMMS The User's Guide", AIMMS B.V., Haarlem, The Netherlands.

Rubin, D. S., Wagner, H. M. (1990) "Shadow Prices: Tips and Traps for Managers and Instructors", INFORMS Journal on Applied Analytics, 20(4), pp. 150-157.

https://doi.org/10.1287/inte.20.4.150 